\title{
Desarrollo de modelos educativos para el aprendizaje de nuevas técnicas endoscópicas: miotomía endoscópica por vía oral (POEM) y elastografía por ultrasonido endoscópico
}

\section{Educational models for learning Peroral Endoscopic Myotomy (POEM) and Endoscopic Ultrasound Elastography}

Martín Alonso Gómez Zuleta, MD,${ }^{*}$ Óscar Fernando Ruiz Morales, MD, ${ }^{2}$ Hernando Marulanda Fernández, MD. ${ }^{3}$

Médico internista y gastroenterólogo. Unidad de gastroenterología y ecoendoscopia, UGEC. Hospital Universitario Nacional. Profesor de Medicina, Universidad Nacional de Colombia.

2 Médico internista y gastroenterólogo. Unidad de gastroenterología y ecoendoscopia, UGEC. Hospital Universitario Nacional. Profesor de Medicina, Universidad Nacional de Colombia.

3 Médico internista. Fellow de gastroenterología, Universidad Nacional de Colombia. Hospital

Universitario Nacional.

*Correspondencia: Martín Alonso Gómez Zuleta martinalonsogomezz@gmail.com

Fecha recibido: $26 / 11 / 18$

Fecha aceptado: 28/01/19

\begin{abstract}
Resumen
Objetivos: presentar el desarrollo de modelos educativos para el aprendizaje de dos técnicas endoscópicas vigentes, ampliamente difundidas y de gran implicación clínica, con la única intención de permitir la adquisición de destrezas básicas y avanzadas a residentes y gastroenterólogos. Materiales y métodos: se idearon dos modelos sencillos, de muy bajo costo, fácilmente reproducibles y reutilizables, con los cuales se logra ejecutar la totalidad de los procedimientos descritos. Además, permiten al operador integrar el desarrollo de habilidades y la adquisición de los conceptos teóricos mínimos requeridos, sin las presiones generadas por el riesgo existente de complicaciones. Resultados: la tendencia actual a nivel mundial se conduce hacia el desarrollo de modelos de enseñanza que aceleren la curva de aprendizaje, así como de procedimientos altamente exigentes y asociados a complicaciones potencialmente graves. Con estos modelos es posible poner a prueba al endoscopista, mediante una evaluación continua y supervisada. Su implementación en unidades de gastroenterología es sencilla, sin la necesidad de una inversión superlativa o el desplazamiento a otros países. Conclusiones: se trata de un gran aporte al desarrollo científico y educativo de nuestro país, ya que la creación y la implementación de nuevas técnicas endoscópicas y su aprendizaje no deben ser asumidos por los pacientes. En este punto, estamos de acuerdo con los conceptos emitidos por diferentes asociaciones médicas respecto a que los cursos teórico-prácticos de corta duración -y en algunas ocasiones virtuales- no constituyen una formación mínima, requerida para lograr la acreditación.
\end{abstract}

\section{Palabras clave}

POEM, elastografía, ecoendoscopia, acalasia, lesiones quísticas, tumores sólidos.

\begin{abstract}
Objectives: This article presents the development of educational models for learning two widespread recent endoscopic techniques which have great clinical implications. Its sole intention is to allow acquisition of basic and advanced skills by residents and gastroenterologists. Materials and methods: Two simple, very low cost, easily reproducible and reusable models were devised. Procedures are fully described in ways that allow the operator to integrate the development of skills and acquisition of the minimum theoretical concepts required without the pressures generated by risks of complications. Results: The current global trend is to develop teaching models that accelerate the learning curve for highly demanding procedures that are associated with potentially serious complications. With these models it is possible to test endoscopists through continuous supervised evaluations. Implementation by gastroenterology units can be done easily without the need for large investments or travel to other countries. Conclusions: This is a great contribution to the scientific and educational development of Colombia since neither development of new endoscopic techniques nor the process of learning how to perform them should put patients at risk. We agree with the ideas of numerous medical associations regarding theoretical-practical courses of short duration even though some virtual sessions, "do not constitute the minimum training required needed for accreditation."
\end{abstract}

Keywords

POEM, elastography, echoendoscopy, achalasia, cystic lesions, solid tumors. 


\section{INTRODUCCIÓN}

Este trabajo propende incentivar el desarrollo de modelos educativos a partir de dos técnicas endoscópicas de gran interés y con una amplia aplicación actual. Nuestro objetivo es permitir la adquisición de destrezas básicas y avanzadas a residentes y gastroenterólogos, así como también definir un método de evaluación sistemática que contribuya a establecer los requerimientos mínimos y objetivos para su práctica.

La implementación de estos modelos constituye, en nuestro criterio, una necesidad, dado que los cursos prácticos de corta duración no representan una formación mínima requerida para acreditarse en dichos procedimientos. En este punto, coincidimos con una gran diversidad de asociaciones médicas.

La explosión en el desarrollo de estas nuevas técnicas endoscópicas requiere un ejercicio continuo de habilidades procedimentales, las cuales no deben realizarse sobre pacientes, ya que las complicaciones derivadas de la impericia son inaceptables (1). Urge entonces el establecimiento de programas estructurados para el aprendizaje en técnicas avanzadas (2), sin la necesidad de una inversión superlativa o el desplazamiento a otros países en su búsqueda (3).

En la actualidad, se cuenta con diferentes tipos de simuladores, dentro de los que se destacan modelos animales, simuladores biológicos y virtuales. Estos simuladores logran acelerar la curva de aprendizaje (entrenamiento) o el mantenimiento de la competencia ante períodos largos sin su ejercicio (reentrenamiento) (4).

Recientemente, la Sociedad Americana de Endoscopia Gastrointestinal (American Society for Gastrointestinal Endoscopy, ASGE) ha conceptuado al respecto y ha considerado que la simulación virtual es inferior frente al modelo animal in vivo y al simulador con piezas orgánicas (5).

\section{LA MIOTOMÍA ENDOSCÓPICA POR VÍA ORAL}

La miotomía endoscópica por vía oral (Per-Oral Endoscopic Myotomy, POEM) es un procedimiento exigente, asociado a complicaciones potencialmente graves (6). Ante la complejidad de esta técnica, la recomendación de las sociedades de endoscopia a nivel mundial es que, luego de dominar procedimientos de terapéutica avanzada, se deben proveer e implementar estrategias de entrenamiento que simulen escenarios clínicos intraprocedimentales, previas a su uso y ejecución en seres humanos (7).

Los modelos que se encuentran actualmente en desarrollo son múltiples y pueden dividirse en biológicos y no biológicos. Dentro de ellos se destacan las espumas, los esófagos y los estómagos explantados de origen animal e incluso animales vivos bajo sedación (8). De estos últimos, es probable que el cerdo sea el modelo animal más apropiado en el desarrollo de habilidades y técnicas para la miotomía endoscópica.

El cerdo muestra ventajas como que cuenta con un esófago más largo, un espacio submucoso más blando y una distribución de las fibras musculares de la unión esofagogástrica variable, lo cual genera un reto adicional durante el entrenamiento (9).

\section{ELASTOGRAFÍA EN ULTRASONIDO ENDOSCÓPICO}

El ultrasonido endoscópico es un procedimiento que ha modificado el quehacer del gastroenterólogo, puesto que ha permitido la integración con diferentes subespecialidades de la medicina. Así, para su interpretación se toman conductas que modifican de forma radical al pronóstico y el tratamiento definitivos (10).

En este contexto, el uso del ultrasonido en Colombia aún no está ampliamente difundido, en vista de los costos de los dispositivos y de que son pocos los centros en los cuales se implementan con una adecuada técnica y alta calidad (11). Por tanto, el entrenamiento de los especialistas en gastroenterología y endoscopia debería incluir en su primera fase la utilización de modelos de aprendizaje, para que mediante una práctica supervisada se alcancen ciertas habilidades antes de la intervención en el paciente y se logren evitar las complicaciones que pudieran surgir durante este proceso (12).

La curva de aprendizaje es larga y sus complicaciones asociadas son, en general, graves. Dentro de estas complicaciones se halla la presencia de perforaciones, sangrados e infecciones (13). Sin embargo, es probable que la complicación más temida por los ecoendoscopistas sea un error en la aproximación diagnóstica, puesto que si ocurre uno de estos casos se corre el riesgo de atentar de forma directa contra la condición del paciente, y de manera indirecta contra el bienestar del médico.

Así bien, se debe asegurar un aprendizaje de las diferentes técnicas (diagnósticas y terapéuticas) sin poner en riesgo la seguridad del paciente (14). Una de las herramientas tecnológicas desarrolladas con este fin, e incluida en los equipos de ultrasonografía de última generación, es la elastografía cuantitativa, la cual permite al operador acercarse a las características estructurales de las alteraciones documentadas, de forma rápida y práctica. Todo ello contribuye a orientar y establecer una probable etiología.

Con todo este panorama, hemos decidido desarrollar un modelo de aplicación elastográfico que permite una aproximación y asimilación del concepto para su implementación adecuada (15). 


\section{MATERIALES Y MÉTODOS}

\section{Elaboración y montaje del modelo POEM}

Para la elaboración de este modelo, se utiliza el bloque de esófago, estómago y segunda porción duodenal, extraído de los cerdos. Se propende, entonces, por dejar un segmento del esófago que en promedio mide $30 \mathrm{~cm}$. Luego de ello, se lava con una solución salina, se retiran los restos de alimentos y se realiza una pequeña incisión a través de la curvatura mayor, con el fin de invaginar la mucosa y lograr una mejor preparación.

Después de seguir estos pasos, se hace énfasis en la unión esofagogástrica y la región subcardial, no solo por el interés en el área de trabajo, sino también porque frecuentemente observamos cómo se acumulan restos de alimentos a este nivel. Seguido de ello, se realiza el cierre de la incisión practicada sobre el cuerpo gástrico con puntos continuos, así como de la segunda porción duodenal para garantizar una adecuada insuflación.

La pieza, en su totalidad, se articula sobre una base de poliestireno expandido (Icopor), que consta de dos láminas cuyo tamaño aproximado es de $50 \times 50 \mathrm{~cm}$. Mientras tanto, en la tapa superior se realiza un corte anatómico de forma que coincida con la disposición y el tamaño del segmento esófago-estómago. Asimismo, sobre la lámina de poliestireno se ubica la placa de la unidad electroquirúr- gica, adherida a la cara posterior del estómago explantado y se le aplica un gel conductor.

En el extremo proximal del esófago se ubica una jeringa de $20 \mathrm{~cm}$ modificada, con el fin de que se disponga en forma de sobretubo y permita el avance del endoscopio con facilidad. Esta jeringa se fija en la porción proximal del esófago, mediante abrazaderas de plástico, las cuales permiten fijar y articular el modelo. Además, se utiliza un guante de látex perforado en el orificio proximal de la jeringa, para que funcione a manera de válvula y se limite la fuga de aire (Figura 1).

Luego de realizar el montaje descrito, se preparan los insumos requeridos dentro del procedimiento. Entre ellos, un endoscopio, una aguja de inyección, solución salina, índigo carmín, una unidad electroquirúrgica modelo ERBE con las configuraciones predeterminadas para la disrupción mucosa y la miotomía, una pinza de coagulación, un bisturí de punta triangular y endoclips (Figuras 2 y 3 ).

\section{Descripción del procedimiento}

$\mathrm{Al}$ iniciar, se realiza una endoscopia diagnóstica y se identifica la distancia a la cual se ubica la unión esofagogástrica. Justo $15 \mathrm{~cm}$ antes de esta, se comienza el procedimiento, elevando la mucosa mediante una inyección de dilución de solución salina e índigo carmín.

Se plantea la posibilidad de realizar abordajes anteriores o posteriores, con el fin de garantizar el desarrollo de las
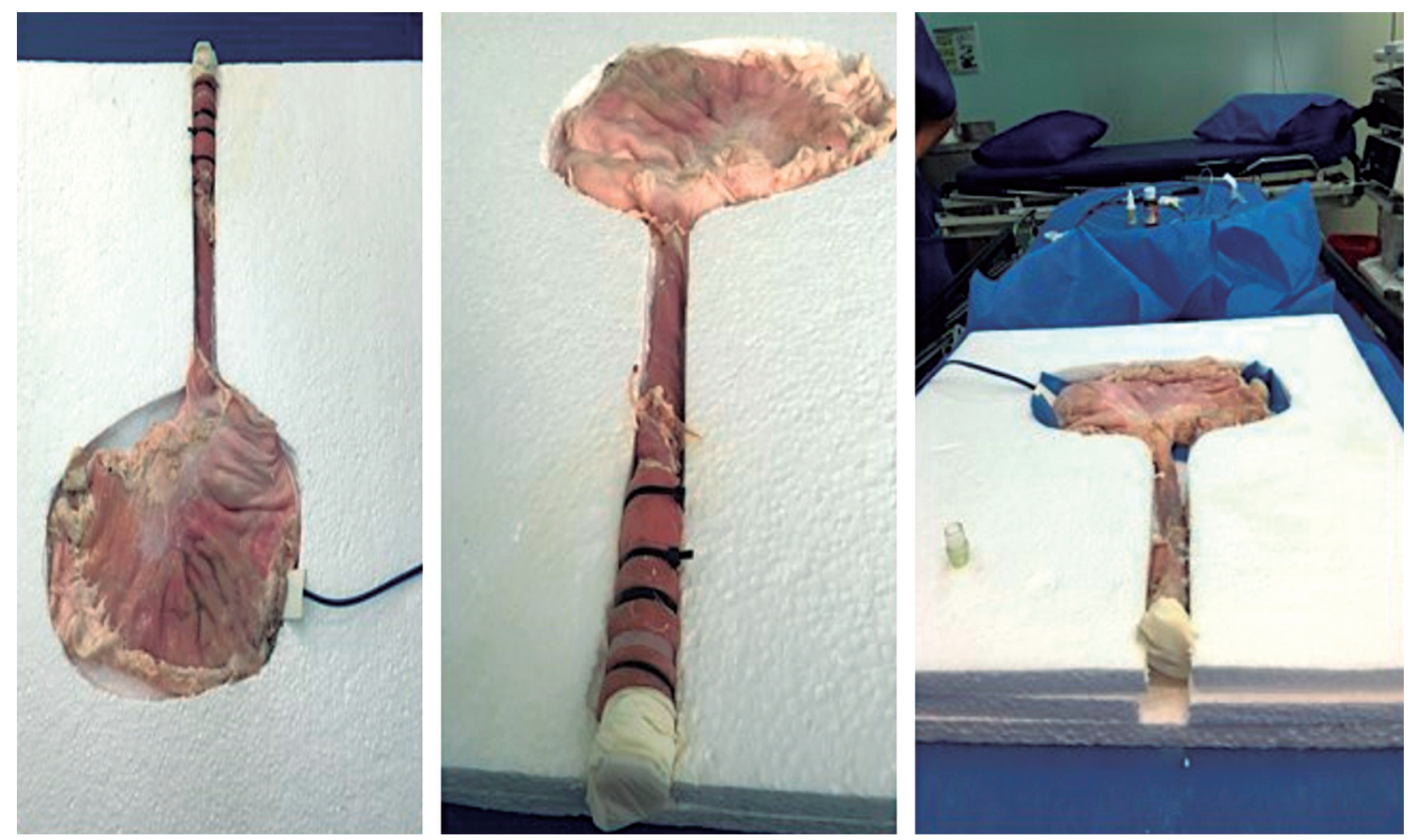

Figura 1. Montaje del modelo POEM. 

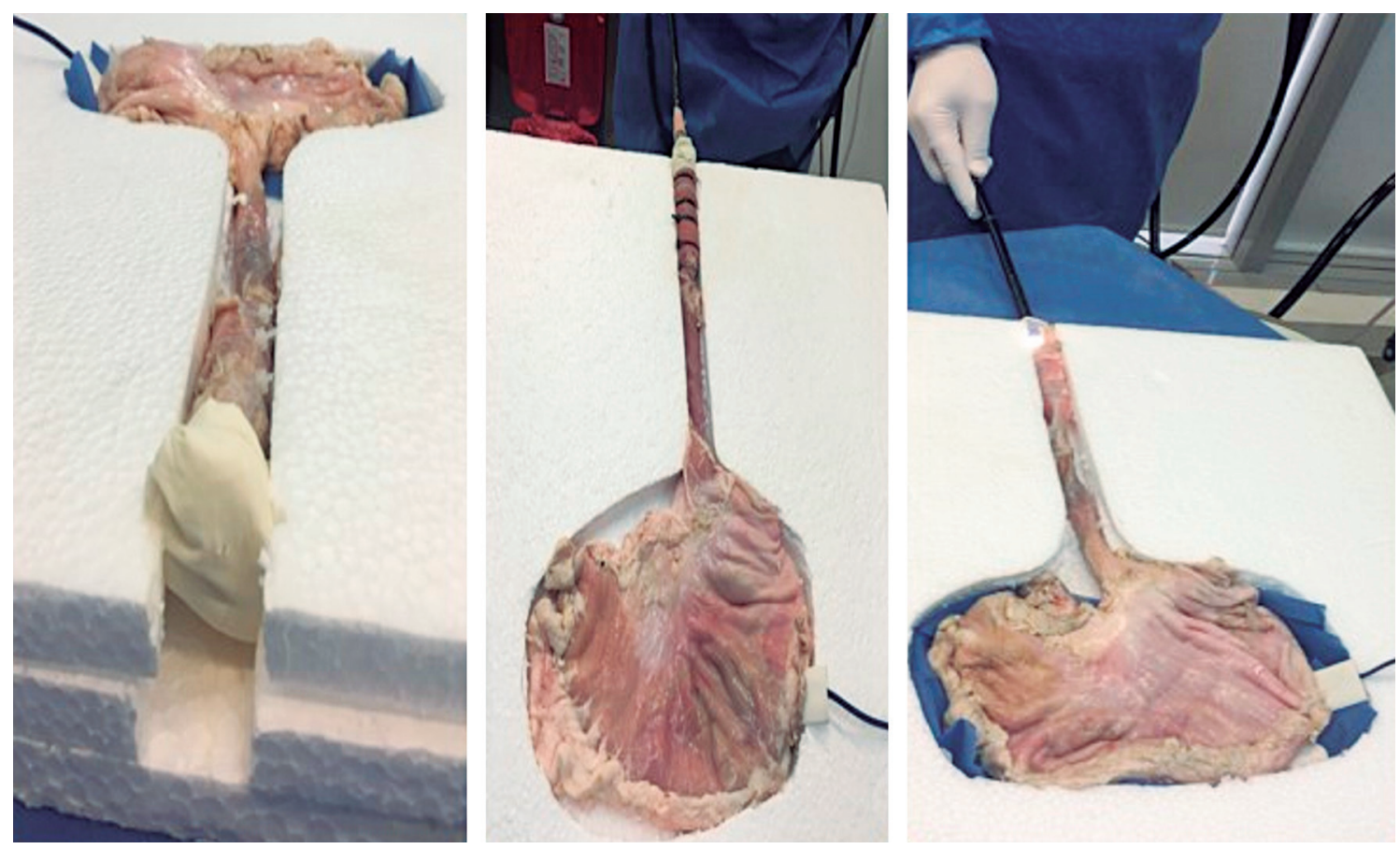

Figura 2. Modelo POEM articulado e inicio del procedimiento.
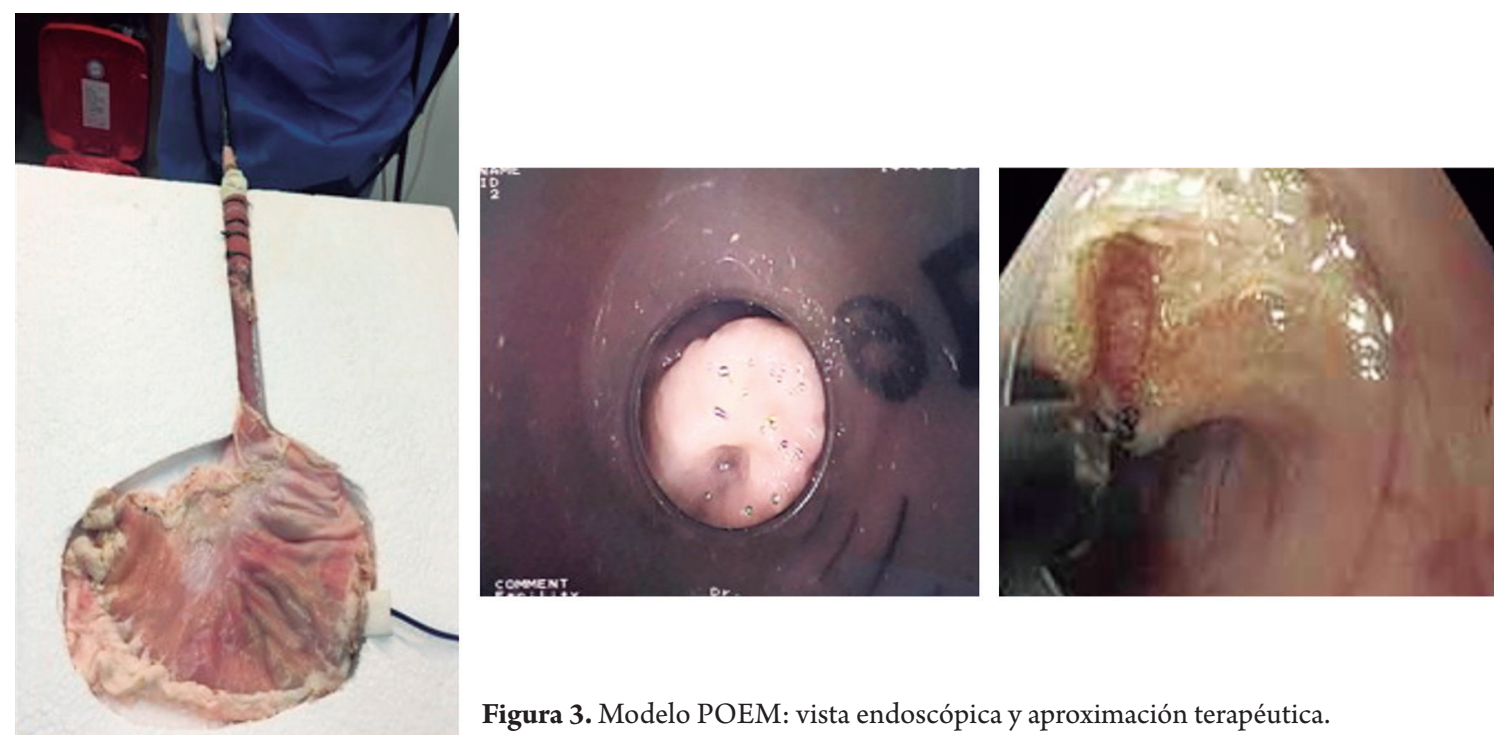

Figura 3. Modelo POEM: vista endoscópica y aproximación terapéutica.

competencias necesarias, ante la posibilidad de múltiples variantes anatómicas encontradas en los pacientes con acalasia.

Luego de escoger el abordaje deseado, se realiza una incisión en la mucosa en sentido transversal de $2 \mathrm{~cm}$ de largo y, posteriormente, se desliza la punta del endoscopio al espacio submucoso, a fin de crear el túnel a partir de una técnica de inyección y disección constante (Figura 4).
Ante la preocupación y las múltiples técnicas descritas para asegurar la profundidad de la inserción, hemos adoptado una modificación a la técnica: se realiza una inyección adicional justo sobre el cardias con índigo carmín, lo cual nos facilita identificar el extremo distal y garantiza la trayectoria deseada con el túnel. Todo ello, sin necesidad del uso de endoscopios accesorios o de retiros repetidos del túnel, lo que podría ampliar el corte mucoso inicial. 

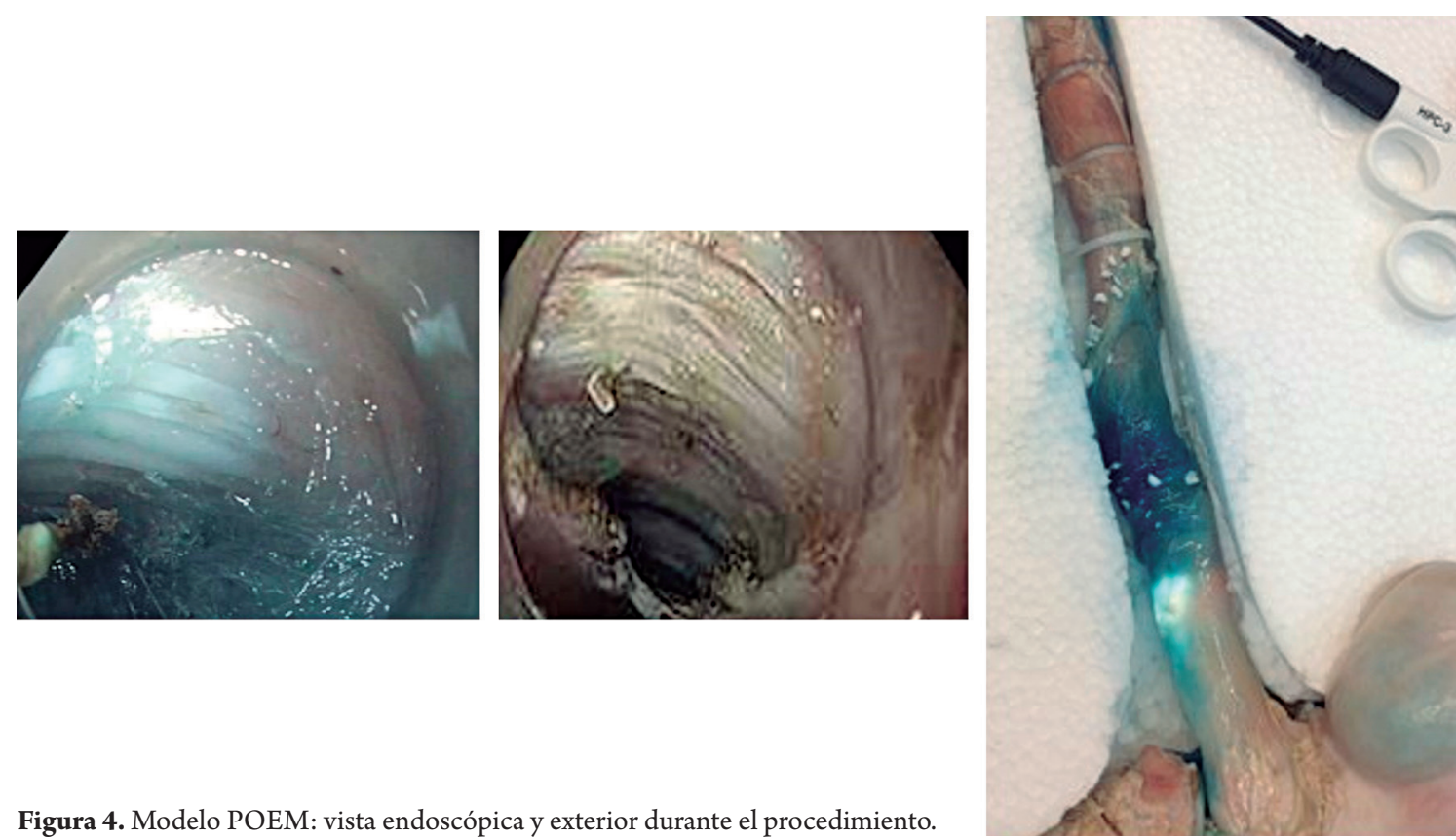

Figura 4. Modelo POEM: vista endoscópica y exterior durante el procedimiento.
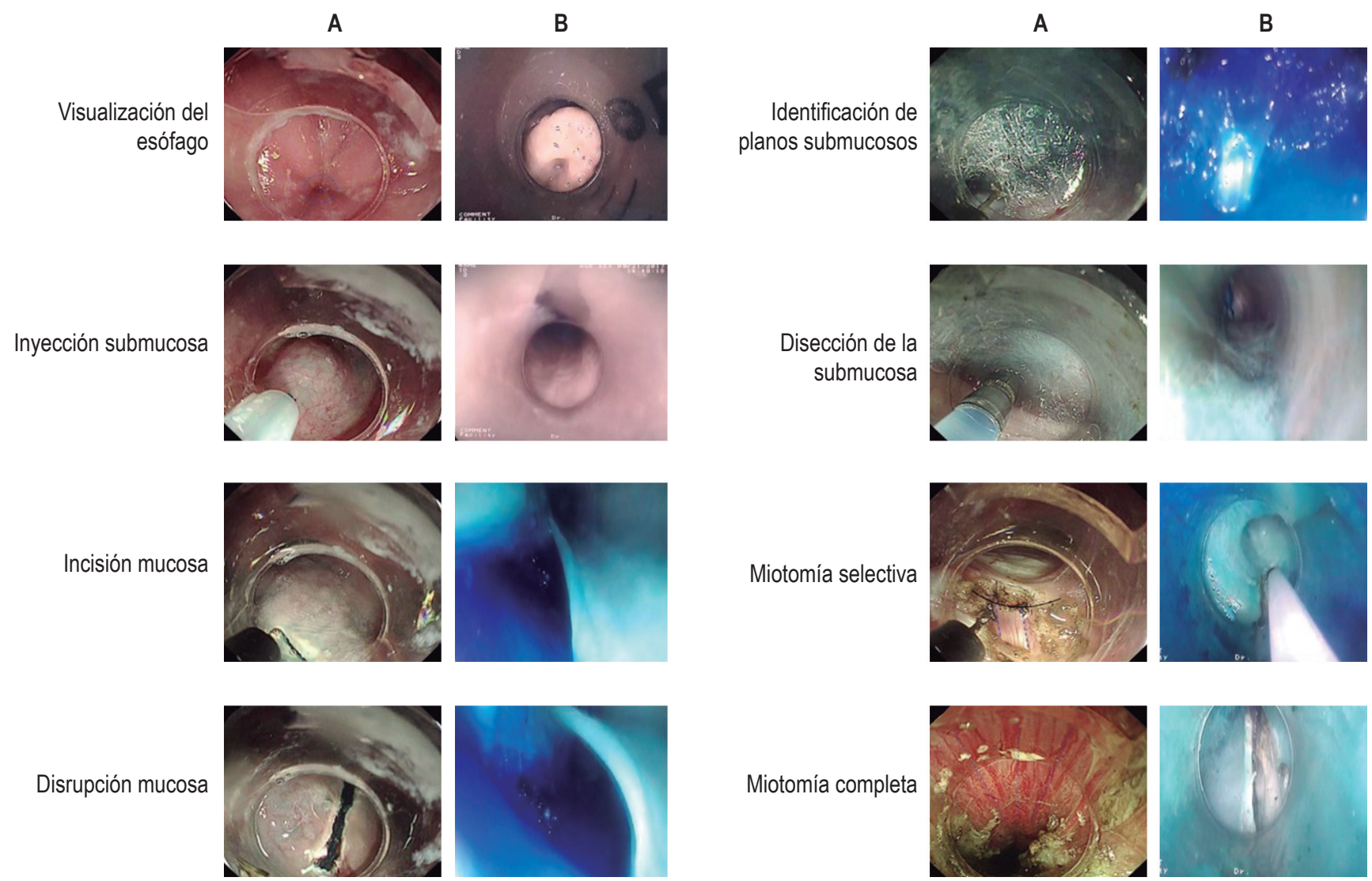

Figura 5. Gráficos comparativos de nuestro modelo (B) con casos in vivo (A). Modificada de la referencia 16. 
La miotomía de las fibras musculares se realiza a partir de la técnica de alzar y cortar hasta $2 \mathrm{~cm}$ de la marca distal o la unión esofagogástrica. Una vez concluida la miotomía, se efectúa el cierre de la incisión mucosa mediante endoclips (16) (Figura 5).

\section{Modelo de elastografía en ultrasonido endoscópico}

\section{Elaboración y montaje}

Para la creación de este modelo, se utilizan materiales como un recipiente plástico de $5 \mathrm{~L}, 400 \mathrm{~g}$ de gelatina, $2 \mathrm{~L}$ de agua, globos de plástico ocupados con aire, agua y aceite y diferentes elementos de distintas consistencias que se introducen dentro del modelo. Entre estos objetos, se emplean pelotas de caucho, uvas, arvejas o cubos de carne, los cuales permiten la evaluación elastográfica.

Así pues, se deben mezclar los $400 \mathrm{~g}$ de gelatina y el agua sobre el recipiente plástico. Cuando la mezcla se solidifique se incluyen los diferentes elementos de apariencia sólida y quística descritos. Luego de ello, el modelo se deja en refrigeración para utilizarlo a la mañana siguiente. Una vez preparado, se esperan $12 \mathrm{~h}$ para su uso. Después de que haya finalizado la jornada de entrenamiento, se refrigera nuevamente para prolongar su uso por 8 días (Figura 6).

\section{Descripción del procedimiento}

El procedimiento se inicia con la ecoendoscopia radial. Antes de empezar esta práctica, se ubica el balón en la punta del ecoendoscopio. Asimismo, el globo puede reemplazarse por globos de piñata, ya que su costo es menor. Posteriormente, se corta el globo por su extremo abierto y luego se fija al transductor con seda dental. Luego de fijar el balón, se introduce el equipo y comienza el aprendizaje, para lo cual es necesario familiarizarse con la consola y demostrar cómo se puede afectar la calidad de la imagen y también de qué forma esta se puede optimizar (ganancias, rangos, contrastes) (Figura 7).

Luego de realizar todo este proceso, se identifican y se evalúan las características de las lesiones que se encuentran (previamente introducidas), las cuales tienen diferentes particularidades ecogénicas. Se hace esta detección y valoración, con el fin de aprender a diferenciar una imagen anecoica (líquida), isoecoica, hipoecoica o hiperecoica.

También se evalúan los diferentes volúmenes (esféricos, ovalados, lineales), lo cual permite realizar una medición de las lesiones y contribuye al aprendizaje en el manejo del cáliper. Estos instrumentos permiten efectuar una medición en dos dimensiones y calcular los volúmenes (Figura 8).

Una vez se hayan completado dichas destrezas, es necesario cambiar al equipo lineal y se muestran las diferencias entre el equipo radial y el lineal. Se identifica, entonces, que la imagen con el equipo radial es de $360^{\circ}$ frente a la generada por la lineal, que es de $160^{\circ}$. De igual forma, se observan las diferencias existentes entre las consolas, el uso del Doppler y la medición de las imágenes.

Al lograr un completo dominio de estas habilidades, se inicia el entrenamiento en la utilidad de la elastografía, a fin de determinar los parámetros relativos a la organización estructural de los tejidos, su consistencia y los cambios de rigidez de los diferentes elementos. Todo ello se basa en la interpretación y el cálculo de los conceptos de Strain Ratio e histograma lineal, lo cual permite una aproximación a la etiología, como en los casos de lesiones neoplásicas frente a las no neoplásicas.
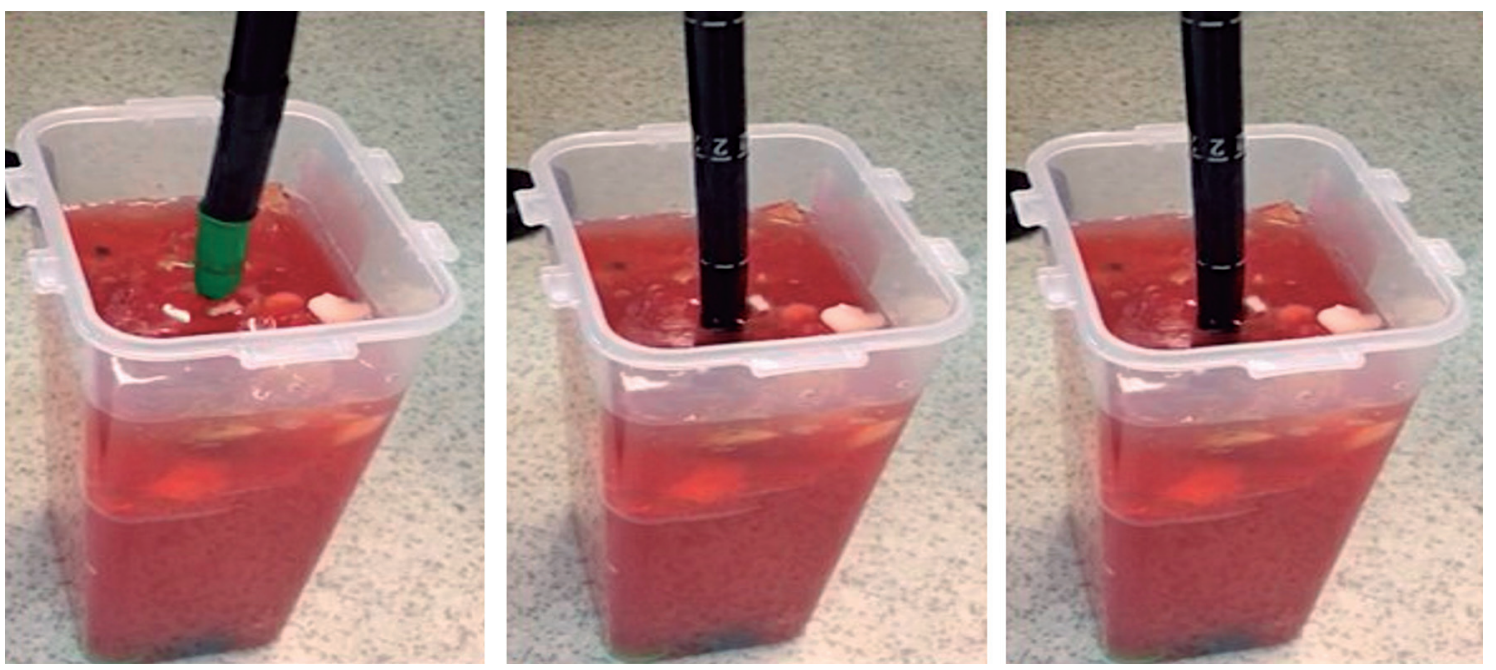

Figura 6. Modelo de elastografía en ultrasonido endoscópico. 


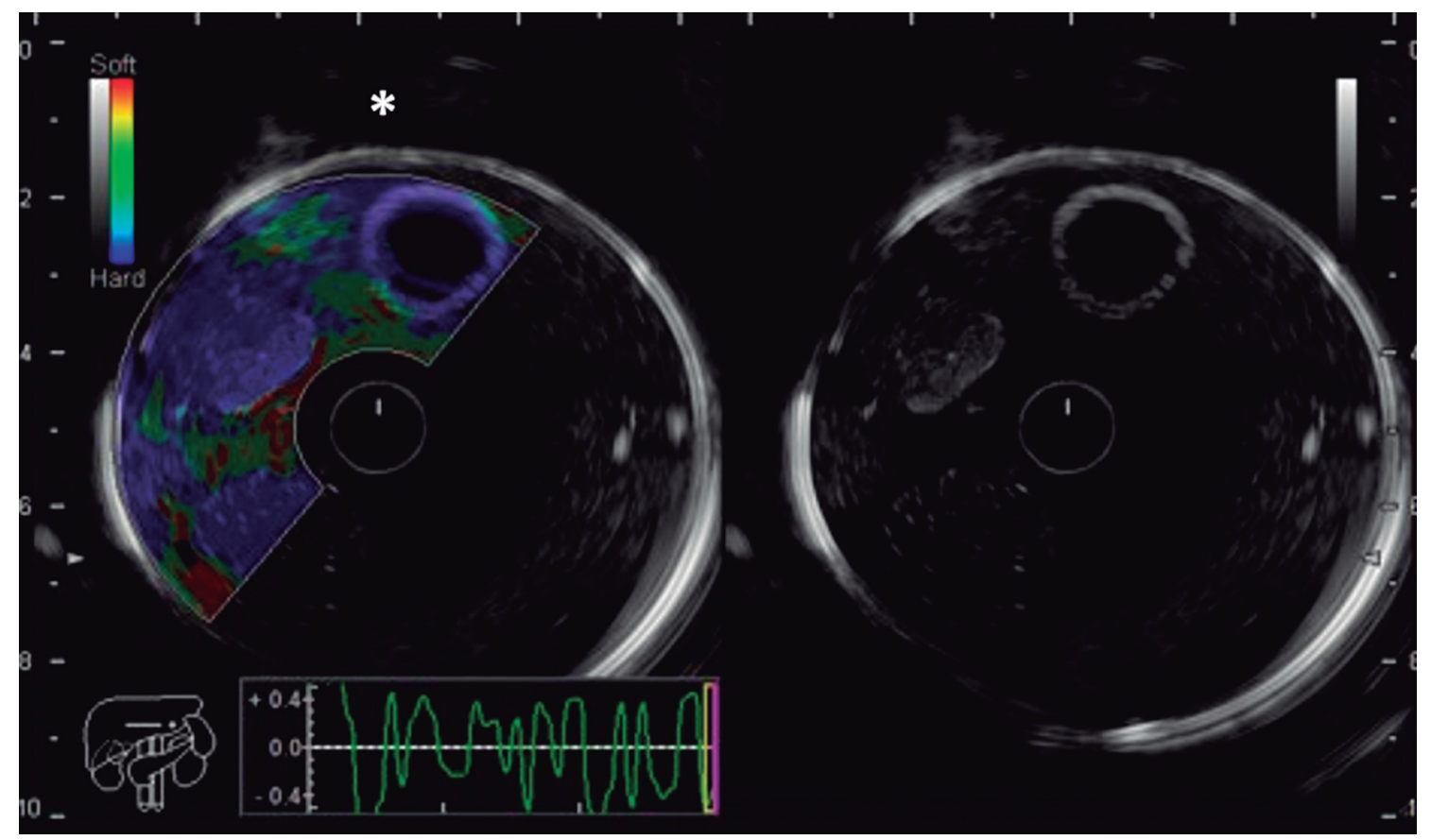

Figura 7. Modelo de elastografía y vista ecoendoscópica: nótense las características ecogénicas de las distintas lesiones (quísticas y sólidas) a la derecha; a la izquierda se demuestra cómo las lesiones quísticas no tienen un patrón elastográfico $\left({ }^{*}\right)$.

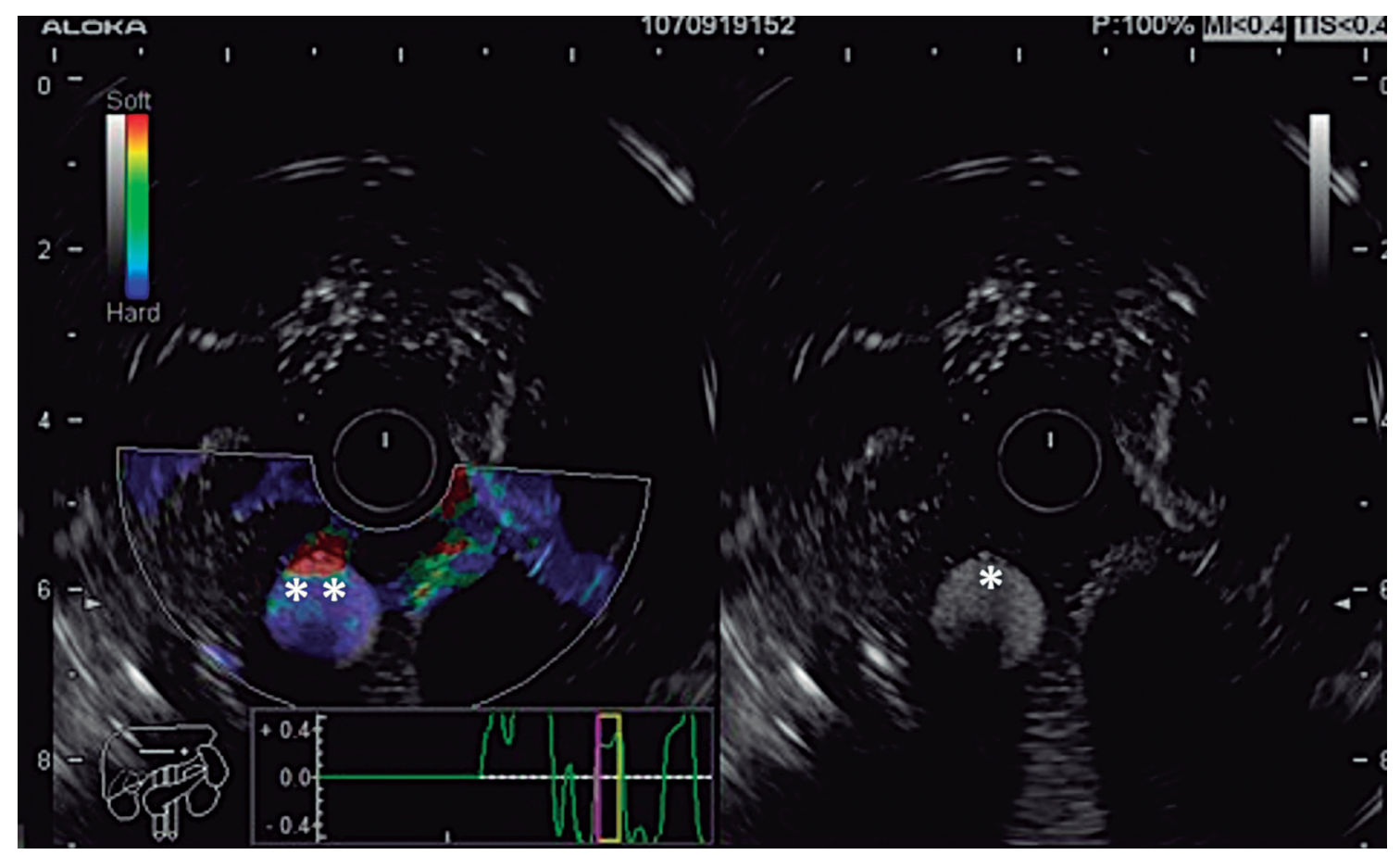

Figura 8. Modelo de elastografía y vista ecoendoscópica: se resaltan las características ecogénicas de una lesión solida a la derecha $(*)$, y su representación elastográfica $\left({ }^{* *}\right)$ 
Entre tanto, la elastografía cuantitativa se basa en la medición de la velocidad de propagación de las ondas transversales de cizallamiento (Shear Wave). Estas ondas se producen en el proceso de compresión de un tejido y su recuperación a la forma original (15). Además, se representan en mapas de calor que varían de azul (tejidos más duros o rígidos) a rojos (tejidos más blandos) (17).

Dentro de este contexto, el valor del Strain Ratio se basa en la relación de la tensión promedio medida en una lesión sólida, en comparación con la tensión promedio de un área similar de tejido sano o adyacente a la lesión (18). La diferencia en la deformación refleja la rigidez relativa de la lesión, tal y como se muestra en la Figura 9. En diversos estudios, un valor de Strain Ratio >20 (más duro) ha sido relacionado con lesiones malignas $(19,20)$.

\section{DISCUSIÓN}

La creación de un tercer espacio (túnel submucoso) ha generado múltiples opciones terapéuticas y diagnósticas para diferentes patologías con resultados favorables y sus indicaciones son cada vez mayores (21). Dentro de ellas se incluyen trastornos funcionales esofágicos como la acalasia, el espasmo difuso esofágico, las alteraciones del vaciamiento gástrico. Además, posibilita las resecciones de tumores subepiteliales esofagogástricos mínimamente invasivos. Por tanto, es fundamental que el endoscopista se familiarice con su técnica y garantice un menor margen de complicaciones derivadas de la intervención per se (22).

De forma intencional, hemos dejado la segunda porción duodenal dentro del modelo, ya que consideramos que esta puede implementarse en la unidad de endoscopia, a fin de realizar la simulación de una POEM gástrica como tratamiento para la gastroparesia (23).

Por otro lado, el modelo de enseñanza de elastografía en ultrasonido endoscópico permite el aprendizaje de los fundamentos básicos de esta interesante técnica de una manera sencilla. Así pues, su uso garantiza una adecuada interacción y el conocimiento de cada uno de los dispositivos de la consola y los equipos. De forma adicional, establece con plenitud la identificación y el detalle de las diferentes características radiológicas de las lesiones, lo que acostumbra a la mano y al ojo para su detección (24).

Se hace fácil el planteamiento de casos clínicos por parte del tutor, previos al enfrentamiento con el modelo, para lo cual se diseña y se elige de forma práctica el tipo de lesión que se va a identificar. Esto permite, además, elaborar un formato de evaluación continua, puesto que si bien el aprendizaje de la técnica es rápido, el gran universo conceptual y la correlación clínica son los aspectos que con-

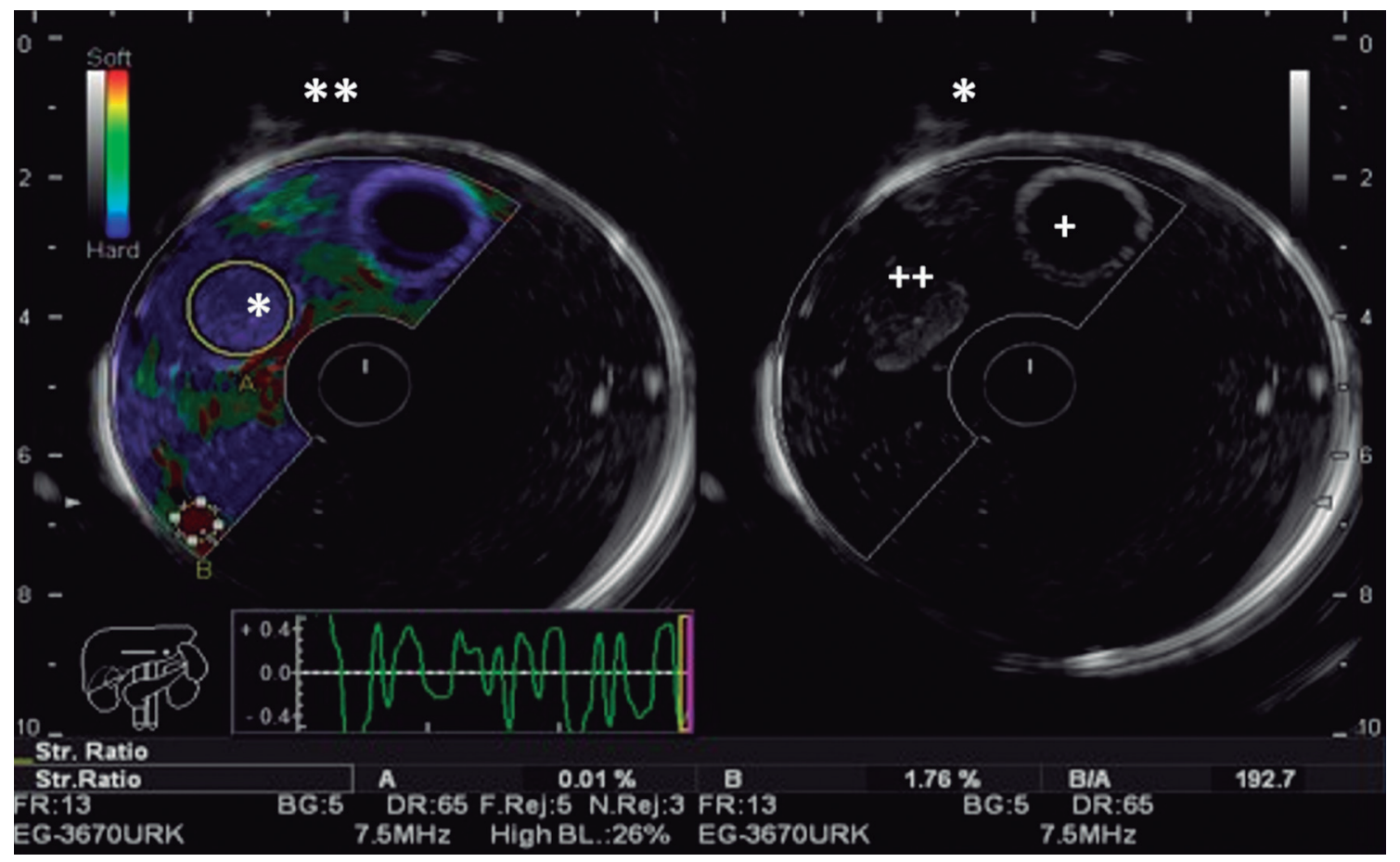

Figura 9. Modelo de elastografía y vista ecoendoscópica: a la derecha, lesión quística (+) y lesión solida (++); a la izquierda se evalúa el patrón elastográfico principalmente azul $\left(^{*}\right)$ y se realiza un cálculo de Strain Ratio (192). 
tribuyen a una adecuada práctica y modifican el umbral terapéutico, sobre la base de los hallazgos elastográficos (25). Dicho factor nos parece fundamental y novedoso, dado que los conceptos excluidos de otros modelos tienen un fin eminentemente diagnóstico en relación únicamente con los hallazgos radiológicos (26-28).

\section{CONCLUSIONES}

Es clara la preocupación que subyace ante la introducción de técnicas diagnósticas y terapéuticas de alto impacto (económico, social, pronóstico, emocional, profesional) como estas. Por tanto, es imperativo que el profesional que las implemente tenga la idoneidad y la experiencia suficientes.

Si bien existen diferentes simuladores con altos detalles tecnológicos, su costo operativo los hace inalcanzables en nuestro medio. Por esto, consideramos nuestro aporte como un impulso fundamental en el desarrollo de las habilidades de gastroenterólogos y residentes en formación.

Los modelos educativos como los nuestros son esenciales en Colombia, puesto que su elaboración tiene un bajo costo. Asimismo, son fácilmente reproducibles, permiten múltiples usos y garantizan el fácil acceso a técnicas especializadas.

\section{REFERENCIAS}

1. Bisschops R, Wilmer A, Tack J. A survey on gastroenterology training in Europe. Gut. 2002;50(5):724-9. http:// dx.doi.org/10.1136/gut.50.5.724

2. McCashland T, Brand R, Lyden E, de Garmo P. The time and financial impact of training fellows in endoscopy. CORI Research Project. Clinical Outcomes Research Initiative. Am J Gastroenterol. 2000;95(11):3129-32. http://dx.doi. org/10.1111/j.1572-0241.2000.03280.x

3. Bini EJ, Firoozi B, Choung RJ, Ali EM, Osman M, Weinshel $\mathrm{EH}$. Systematic evaluation of complications related to endoscopy in a training setting: A prospective 30-day outcomes study. Gastrointest Endosc. 2003;57(1):8-16. https:// doi.org/10.1067/mge.2003.15

4. Cohen J, Thompson CC. The next generation of endoscopic simulation. Am J Gastroenterol. 2013 Jul;108(7):1036-9. https://doi.org/10.1038/ajg.2012.390

5. Varadarajulu S, Christein JD, Wilcox M. 298 Endoscopic Transmural Drainage of Pancreatic Fluid Collections (PFCs) in 200 Consecutive Patients: An Assessment of Outcomes. 2011;73(4):AB122. https://doi.org/10.1016/j. gie.2011.03.038

6. AbouHashem Y, Dayal M, Savanah S, Štrkalj G. The application of 3D printing in anatomy education. Med Educ Online. 2015;20:29847. https://doi.org/10.3402/meo.v20.29847

7. Eleftheriadis $\mathrm{N}$, Inoue $\mathrm{H}$, Ikeda $\mathrm{H}$, Onimaru M, Maselli R, Santi G. Submucosal tunnel endoscopy: Peroral endoscopic myotomy and peroral endoscopic tumor resection. World
J Gastrointest Endosc. 2016;8(2):86-103. https://doi. org/10.4253/wjge.v8.i2.86

8. Li QL, Zhou PH. Perspective on peroral endoscopic myotomy for achalasia: Zhongshan experience. Gut Liver. 2015;9(2):152-8. https://doi.org/10.5009/gnl14227

9. Onimaru $M$, Inoue $H$, Ikeda $H$, Sato $C$, Sato $H$, Phalanusitthepha $\mathrm{C}$, et al. Greater curvature myotomy is a safe and effective modified technique in peroral endoscopic myotomy (with videos). Gastrointest Endosc. 2015;81(6):1370-7. https://doi.org/10.1016/j. gie.2014.11.014

10. Onimaru M, Inoue H, Ikeda H, Yoshida A, Santi EG, Sato $\mathrm{H}$, et al. Peroral endoscopic myotomy is a viable option for failed surgical esophagocardiomyotomy instead of redo surgical Heller myotomy: a single center prospective study. J Am Coll Surg. 2013;217(4):598-605. https://doi. org/10.1016/j.jamcollsurg.2013.05.025

11. Eleftheriadis N, Protopapas A, Katsogridakis J, Hatzitolios AI. Successful peroral endoscopic myotomy for radical treatment of sigmoid-type esophageal achalasia by Greek gastroenterologists. Ann Gastroenterol. 2014;27(4):430-431.

12. Li QL, Chen WF, Zhou PH, Yao LQ, Xu MD, Hu JW, et al. Peroral endoscopic myotomy for the treatment of achalasia: a clinical comparative study of endoscopic full-thickness and circular muscle myotomy.J Am Coll Surg. 2013;217(3):44251. https://doi.org/10.1016/j.jamcollsurg.2013.04.033

13. Sedlack RE, Kolars JC. Computer simulator training enhances the competency of gastroenterology fellows at colonoscopy: results of a pilot study. Am J Gastroenterol. 2004;99(1):33-7. https://doi.org/10.1111/j.1572-0241.2004.04007.x

14. Gómez MA. Utilidad de un modelo para el entrenamiento del ultrasonido endoscópico radial y lineal. Rev Col Gastroenterol. 2007;22:104-110.

15. Nightingale K, Bentley R, Trahey G. Observations of tissue response to acoustic radiation force: opportunities for imaging. Ultrason Imaging. 2002;24(3):129-38. https://doi. org/10.1177/016173460202400301

16. Grimes KL, Inoue H. Per Oral Endoscopic Myotomy for Achalasia: A Detailed Description of the Technique and Review of the Literature. Thorac Surg Clin. 2016;26(2):14762. https://doi.org/10.1016/j.thorsurg.2015.12.003

17. Nightingale K, McAleavey S, Trahey G. Shear-wave generation using acoustic radiation force: in vivo and ex vivo results. Ultrasound Med Biol. 2003;29(12):1715-23. https://doi.org/10.1016/j.ultrasmedbio.2003.08.008

18. Bercoff J, Tanter M, Fink M. Supersonic shear imaging: a new technique for soft tissue elasticity mapping. IEEE Trans Ultrason Ferroelectr Freq Control. 2004;51(4):396-409. https://doi.org/10.1109/TUFFC.2004.1295425

19. Sandrin L, Fourquet B, Hasquenoph JM, Yon S, Fournier C, Mal F, Christidis C, Ziol M, Poulet B, Kazemi F, Beaugrand M, Palau R. Transient elastography: a new noninvasive method for assessment of hepatic fibrosis. Ultrasound Med Biol. 2003;29(12):1705-13. https://doi.org/10.1016/j. ultrasmedbio.2003.07.001 
20. Sandrin L, Tanter M, Catheline S, Fink M. Shear modulus imaging with 2-D transient elastography. IEEE Trans Ultrason Ferroelectr Freq Control. 2002;49(4):426-35. https://doi.org/10.1109/58.996560

21. Harewood GC, Wiersema MJ, Nelson H, Maccarty RL, Olson JE, Clain JE, et al. A prospective, blinded assessment of the impact of preoperative staging on the management of rectal cancer. Gastroenterology. 2002;123(1):24-32. https://doi.org/10.1053/gast.2002.34163

22. Chang KJ, Nguyen P, Erickson RA, Durbin TE, Katz KD. The clinical utility of endoscopic ultrasound-guided fineneedle aspiration in the diagnosis and staging of pancreatic carcinoma. Gastrointest Endosc. 1997;45(5):387-93. https://doi.org/10.1016/S0016-5107(97)70149-4

23. Hochberger J, Maiss J, Magdeburg B, Cohen J, Hahn EG. Training simulators and education in gastrointestinal endoscopy: current status and perspectives in 2001. Endoscopy. 2001;33(6):541-9. https://doi.org/10.1055/s-2001-14972
24. Silver B, Metzger TS, Matalon TA. A simple phantom for learning needle placement for sonographically guided biopsy. AJR Am J Roentgenol. 1990;154(4):847-8. https:// doi.org/10.2214/ajr.154.4.2107686

25. Burmester E, Leineweber T, Hacker S, Tiede U, Hütteroth TH, Höhne KH. EUS Meets Voxel-Man: three-dimensional anatomic animation of linear-array endoscopic ultrasound images. Endoscopy. 2004;36(8):726-30. https://doi. org/10.1055/s-2004-825669

26. Fusaroli P, Caletti G. Endoscopic ultrasonography. Endoscopy. 2003;35(2):127-35. https://doi. org/10.1055/s-2003-37010

27. Classen M, Ruppin H. Practical Endoscopy Training Using a New Gastrointestinal Phantom. Endoscopy 6 (1974) 127131. https://doi.org/10.1055/s-0028-1098609

28. Qiao W, Bai Y, Lv R, Zhang W, Chen Y, Lei S, et al. The effect of virtual endoscopy simulator training on novices: a systematic review. PLoS One. 2014;9(2):e89224. https://doi. org/10.1371/journal.pone.0089224 